\title{
COMMENT Pediatric research today and tomorrow post-COVID-19
}

\author{
Tina L. Cheng $\mathbb{D}^{1,2}$, Sherin U. Devaskar ${ }^{3}$ and on behalf of the Pediatric Policy Council
}

Pediatric Research (2021) 90:13-15; https://doi.org/10.1038/s41390-021-01520-8

The COVID-19 pandemic is a historical turning point with farreaching effects on children, families, and communities. The pandemic has highlighted the tremendous importance of science in (1) epidemiology in describing and tracking the pandemic and disparities, (2) discovery in understanding the virus along with host responses, (3) clinical translation in developing vaccines and treatments and their clinical trials, and (4) public health in developing and implementing preventive interventions, health promotion, and ensuring health equity. Recognizing the crucial role played by science in charting our path forward, how will pediatric research change?

In this issue of Pediatric Research, Levy-Mendelovich et al. ${ }^{1}$ examine trends in the pediatric literature over the past two decades pre-COVID using text mining tools. Upon review of abstracts from pediatric journals, they noted a shift in the published literature with a greater proportion of studies categorized as epidemiologic, psychology, and psychiatry research.

While the epidemiologic study of the distribution, patterns, and determinants of health and disease in populations is critically important, especially during this pandemic, it is also essential to move beyond a mere description of poor health to address etiology and solutions, thereby making an impactful difference. The COVID-19 pandemic has not only demonstrated the necessity of epidemiologic tracking of disease(s) to guide urgent prevention and management strategies, but has highlighted the importance of the entire translational science spectrum of the National Center for Advancing Translational Sciences (NCATS) (Fig. 1). ${ }^{2}$ The NCATS definition of translation has been on full display during the pandemic: "The process of turning observations in the laboratory, clinic and community into interventions that improve the health of individuals and the public-from diagnostics and therapeutics to medical procedures and behavioral changes".2

A misconception is that this translation follows linear, directional steps from basic science (T0) to preclinical research (T1) to clinical research (T2) to clinical implementation (T3) and to public health (T4). ${ }^{3}$ Instead, each of these steps is often "complementary, interdependent and mutually informative".2,4 The pandemic has emphasized the need for rapid translation, the critical mutuality and interconnections across the spectrum, and the iterative process needed over time. For instance, as epidemiologic virus surveillance has found new variants, new discovery research has been required, with potential new vaccines, new clinical trials, and new dissemination plans. Beyond description, there is a continuing need to use tools of science including randomized trials and fundamental etiologic research. Understanding the mechanisms of why children have been less seriously affected by acute COVID-19 infection compared to adults and why some children develop Multisystem Inflammatory Syndrome in Children or become "long haulers" are sorely needed.
Figure 1 appropriately emphasizes the importance of patient/ subject and family involvement at the center of translational efforts, necessary in informing and directing researchers' creativity while simultaneously empowering those directly affected. It is heartening that the Levy-Mendelovich et al. study found an increased number of pediatric psychology and psychiatry publications in the literature considering growing mental, emotional, and behavioral child and family needs pre-COVID. ${ }^{5}$ The pandemic has exacerbated the pre-existing mental health crisis and calls for further investigations in this critically essential field. ${ }^{6}$

Developing effective strategies to address the pandemic requires that the patient and community be at the center of all scientific efforts. This is necessary to understand and overcome the roots of the unacceptable racial/ethnic and socioeconomic disparities that exist in COVID-19 cases, hospitalizations and deaths highlighting the urgent need to ensure health equity. ${ }^{7}$ It also includes participation in clinical research and vaccine trials that inform strategies to encourage vaccine acceptance and vaccine access. Over one decade ago, a technical report from the American Academy of Pediatrics noted many descriptions of racial/ethnic and socioeconomic health disparities in the literature and the dearth of community-informed intervention research. ${ }^{8}$

While review articles contributed to the majority of pediatric publications analyzed in the article by Levy-Mendelovich et al., they include many multicenter clinical studies. Collaborative combination of data acquired across multiple sites offers greater power, generalizability, and learning with a readiness towards implementation. Rapid multisite collaborations during the pandemic have been key to the development of effective clinical preventive and interventional strategies. ${ }^{9}$

In addition, cross-disciplinary and multisector expertise in research involving the education, social services, and juvenile justice sectors will further research inquiry and address our shared vision and goals of healthy children who are ready to learn and play while achieving their optimal trajectory to productive adulthood. The pandemic has shown the interdependence of various sectors that were previously considered disparate in ensuring optimal child health such as enhanced collaboration on school openings, camps, and daycare. Crosstalk between various disciplines takes us from scratching the descriptive surface to the heart of the problem, creating in-roads into targeted interventions and the much-needed ultimate solutions.

While the Levy-Mendolovich study focused on pediatric journals and yesterday's science, future research must go beyond pediatric journals and incorporate a family and life course approach. The pandemic has increased awareness of the influence of family dynamics on disease transmission and the impact of child health

\footnotetext{
${ }^{1}$ Department of Pediatrics, University of Cincinnati College of Medicine, Cincinnati, OH, USA; ${ }^{2}$ Cincinnati Children's Hospital Medical Center, Cincinnati, OH, USA and ${ }^{3}$ Department of Pediatrics, David Geffen School of Medicine at University of California, Los Angeles, CA, USA

Correspondence: Tina L. Cheng (Tina.Cheng@cchmc.org)
}

Received: 18 March 2021 Accepted: 21 March 2021

Published online: 13 April 2021 


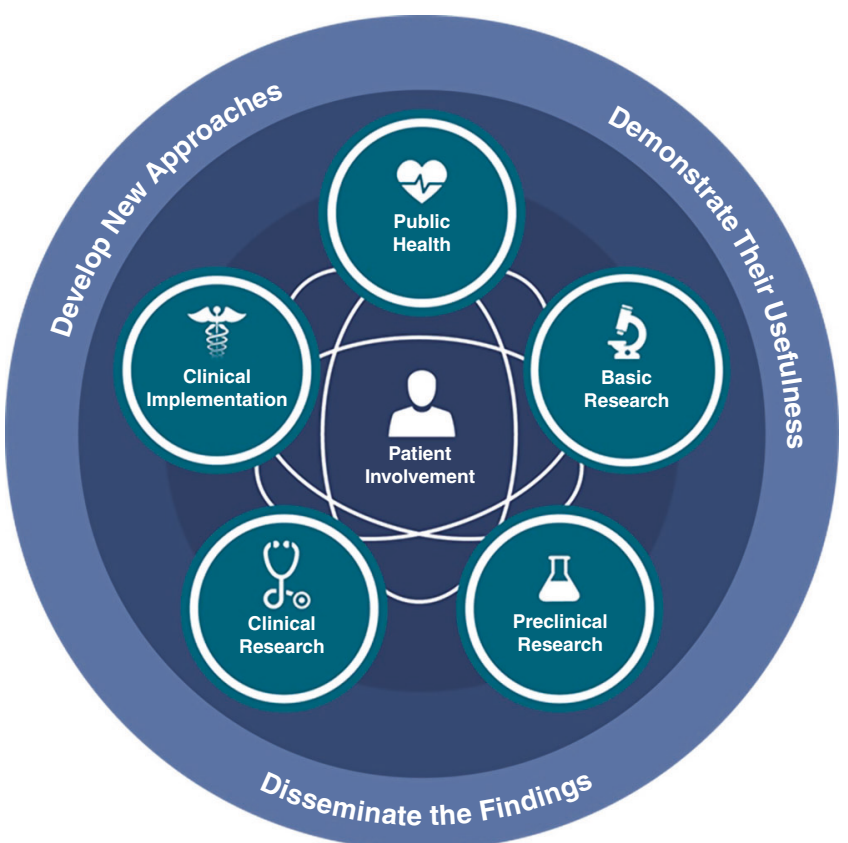

Fig. 1 The Translational Science Spectrum. The National Center for Advancing Translational Sciences (NCATS) - one of 27 Institutes and Centers at the National Institutes of Health $(\mathrm{NIH})$ - illustrates "the nonlinear, multidirectional process of scientific research from knowledge about the biological basis of health and disease to delivery of interventions that improve the health of individuals and the public".

and school attendance on psychological wellbeing as well as adult health and productivity. It is known that the physical and mental health of mothers can have an enduring effect on the physical and mental health of their infants. Healthy children require healthy parents. Conversely, healthy, working parents are influenced by the health and caring of their children. Parents cannot go to work if their children are not in daycare or school or if their children are ill. Pediatric research and policy (e.g., parental leave, living wage) must focus on the family unit.

Further, burgeoning research supports child health or disease forming the foundation of later health under the auspices of "developmental origins of adult health and disease". ${ }^{10,11}$ Increasingly genetics/epigenetics and early detection of various biomarkers will become the mainstay of predicting pediatric diseases and provide opportunities for intervention prior to the emergence of disabling symptomatology. Family and life course investigations are central to the future of science, bringing to bear discoveries and innovation on population health.

Finally, the Levy-Mendelovich article noted the global nature of pediatric research. The pandemic has clearly shown that we are all part of a global society requiring global solutions. Dissemination and implementation research is needed to ensure scientific discoveries, like vaccines, have no barriers, reaching everyone. Global health equity is not only a moral imperative but is of benefit to all of us. While it is fortunate that the incidence of children being affected is less and generally the acute morbidity and mortality from COVID-19 compared to adults is also lower, the impact on children and families has been devastating. ${ }^{12}$ With the decreasing birth rate due to the pandemic and lower rates of hospitalizations and death among children, there is the dire possibility that pediatric research will be given lower priority. For instance, COVID-19 vaccine clinical trials including children and pregnant and lactating women have been limited and more robust studies are urgently needed..$^{13,14}$ Recognizing children are our most precious resource and our future workforce, ensuring their wellbeing and addressing early antecedents to adult disease should be of utmost importance. Investment in prenatal and pediatric research supports prevention at the very beginning of life's trajectory, expanding health span and lifespan. As Frederick Douglas has said, "It is easier to build strong children than to repair broken men". While children constitute $9 \%$ of US healthcare spending ${ }^{15}$ they represent $100 \%$ of our future.

The pandemic has taught us many lessons including the value of science. Let us not lose this moment of the general public's recognition that science is the key driver behind all innovative preventive and therapeutic interventions, paving the road to health by mitigating disease. Looking post-pandemic, pediatric research must strive to address the entire translational spectrum (from basic science to clinical science to population health science and policy), while continuing to be patientcentered and equity-focused, multisite, cross-disciplinary, and with global reach. We must seize the moment to recalibrate our thought processes, training, and strategy on behalf of children and families. Advocacy for pediatric research that is family and life course-oriented offers promise for a better and equitable tomorrow.

\section{DISCLAIMER}

No financial assistance was received to support this paper. This work is original, not previously published, and not submitted for publication or consideration elsewhere.

\section{AUTHOR CONTRIBUTIONS}

T.L.C.: conceptualized and wrote the manuscript, revised each draft, and approved the final manuscript. S.U.D.: wrote portions of the manuscript, revised each draft, and approved the final manuscript.

\section{ADDITIONAL INFORMATION}

Competing interests: The authors declare no competing interests.

Publisher's note Springer Nature remains neutral with regard to jurisdictional claims in published maps and institutional affiliations.

\section{REFERENCES}

1. Levy-Mendelovich, S. et al. Pediatric literature trends: high level analysis using text-mining. Pediatr. Res. (in the press).

2. National Center for Advancing Translational Science. Translational Science Spectrum. https://ncats.nih.gov/translation/spectrum (2021).

3. Committee to Review the Clinical and Translational Science Awards Program at the National Center for Advancing Translational Sciences; Board on Health Sciences Policy; Institute of Medicine. In The CTSA Program at NIH: Opportunities for Advancing Clinical and Translational Research (eds Leshner, A. I. et al.) (National Academies Press, 2013).

4. Austin, C. P. Translating translation. Nat. Rev. Drug Discov. 17, 455-456 (2018).

5. National Academies of Sciences, Engineering, and Medicine. Fostering Healthy Mental, Emotional, and Behavioral Development in Children and Youth: A National Agenda (The National Academies Press, 2019).

6. Leeb, R. T. et al. Mental Health-Related Emergency Department visits among children aged $<18$ years during the COVID-19 pandemic - United States, January 1-October 17, 2020. Morb. Mortal. Wkly Rep. 69, 1675-1680 (2020).

7. Centers for Disease Control and Prevention. COVID-19 Racial and Ethnic Health Disparities. https://www.cdc.gov/coronavirus/2019-ncov/community/healthequity/racial-ethnic-disparities/index.html (2021).

8. Flores, G. and the American Academy of Pediatrics Committee on Pediatric Research. Technical report: racial and ethnic disparities in the health and health care of children. Pediatrics 125, e979-e1021 (2010).

9. Lane, H. C. \& Fauci, A. S. Research in the context of a pandemic. N. Engl. J. Med. 384, 755-757 (2021). 
10. Halfon, N. \& Hochstein, M. Life course health development: an integrated framework for developing health, policy, and research. Milbank Q. 80, 433-479 (2002).

11. Cheng, T. L. \& Solomon, B. S. Translating Life Course Theory to clinical practice to address health disparities. Matern. Child Health J. 18, 389-395 (2014).

12. Cheng, T. L., Moon, M. \& Artman, M., Pediatric Policy Council. Shoring up the safety net for children in the COVID-19 pandemic. Pediatr. Res. 88, 349-351 (2020).
13. Adhikari, E. H. \& Spong, C. Y. COVID-19 vaccination in pregnant and lactating women. JAMA https://doi.org/10.1001/jama.2021.1658 (2021).

14. Anderson, E. J. et al. Warp speed for COVID-19 vaccines: why are children stuck in neutral? Clin. Infect. Dis. 18, https://doi.org/10.1093/cid/ciaa1425 (2020).

15. Hahn, H. et al. Kids' Share 202 Report on Federal Expenditures on Children Through 2019 and Future Projections (Urban Institute, 2020). 